\title{
The Theory and Experimental Study of the Self-Excited Oscillation Pulsed Jet Nozzle (Pipeline Pulsed Flow Generator)
}

\author{
Shiqiang Lai, Zhengfang Liao* \\ College of Materials Science and Engineering, Chongqing University, Chongqing, China. \\ Email: ${ }^{*}$ liaozf@263.net \\ Received June $14^{\text {th }}, 2013$; revised August $3^{\text {rd }}, 2013$; accepted August 21 ${ }^{\text {st }}, 2013$ \\ Copyright (C) 2013 Shiqiang Lai, Zhengfang Liao. This is an open access article distributed under the Creative Commons Attribution \\ License, which permits unrestricted use, distribution, and reproduction in any medium, provided the original work is properly cited.
}

\begin{abstract}
In this paper, the basic theories of the Self-excited Oscillation Pulsed Jet Nozzle (SEOPJN) invented by the authors are introduced. Then, some experimental results are shown. According to the results, using tricorn bits assembled the SEOPJN to drill oil well, the rate of penetration (ROP) increases by $8 \%-77 \%$, and the rate of the footage for tricorn bit increases by $6.7 \%-44 \%$. Although the test was conducted in the water, good result was got in nature gas transportation. The volume of gas transportation could be increased by the Self-excited Oscillation Pulsed generator while the gas pressure drop could be decreased, since it significantly reduced the pressure loss during gas transportation.
\end{abstract}

Keywords: Self-Excited Oscillation Pulsed Jet; Nozzle; Oil Well; Natural Gas

\section{Introduction}

With the rapid development of industrialization, the demand for gasoline and natural gas is booming nowadays. Therefore how to raise the output and reduce the pressure loss in transportation becomes an essential topic. Based on 30 years of research and experiment, the author thinks that pulsed flow is one of the best solutions for these problems. The results from experiment show that pulsed flow can significantly increase the speed of drilling oil well. The pulsed flow can turn the flow in pipes into fluctuation motion (like sliding friction turns to roll friction in the mechanical drive to reduce friction loss) The afore mentioned idea has passed though the test in the nature gas transmission to get demonstration.

\section{The Structure of the Self-Excited Oscillation Pulsed Jet Nozzle (SEOPJN) $[1,2]$}

Just as shown in Figure 1, the SEOPJN consists of three parts. The first part is named as upstream nozzle, and the second part is named as self-excited oscillation cavity. The third part is named as downstream nozzle.

\footnotetext{
${ }^{*}$ Corresponding author.
}

\section{The Operating Mechanism of SEOPJN [3]}

According to the theory of boundary layer and vortex, when jet or shearing flow flows through self-excited oscillation cavity, some vortex waves are generated at separating zone (Figure 2). Due to the un-stability of shearing layer, it is possible that the vortex waves with some certain frequencies are amplified when they flow downward. So, a series of discrete vortex rings are formed in shearing layer. With these discrete vortex rings transmitting downward, when they come to impinging edge and react with impinging edge, pressure pulse waves are generated. The waves are reflected and transmitted upward. If the phase position of the waves trans-

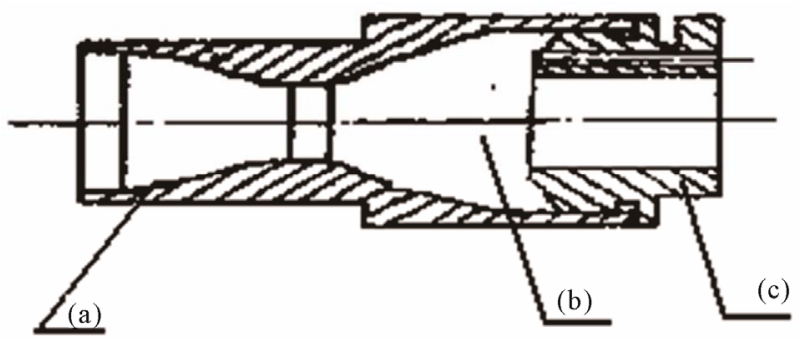

Figure 1. Structure of SEOPJN. (a) Upstream nozzle; (b) Self-excited oscillation cavity; (c) downstream nozzle. 


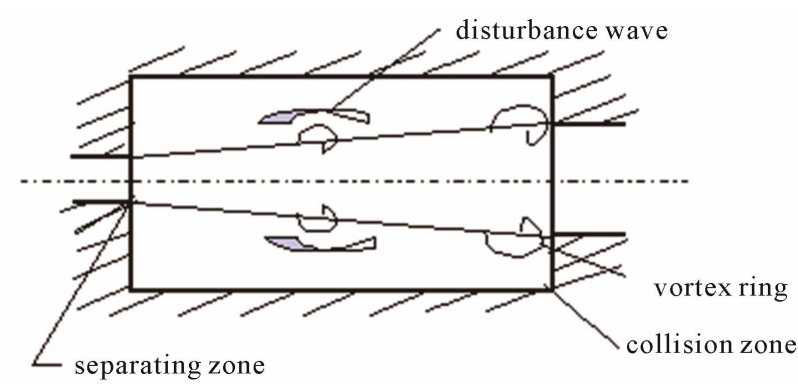

Figure 2. Operating mechanism of SEOPJN.

mitting downward and the phase position of the waves transmitting upward are reverse, these waves are added repeatedly to form new waves whose amplitude are larger. With the process going on, self-excited oscillation pulsed jet is generated.

\section{Theoretical Analysis of Self-Excited Oscillation Pulsed Jet [4,5]}

In self-excited oscillation cavity, only when some conditions are satisfied, intensive self-excited oscillation waves can be generated. The first condition is that some vortex disturbance waves must be generated at the exit of up-stream nozzle. The second condition is that the amplitude of the disturbance waves with some certain frequencies must be amplified. The third condition is that the disturbance waves and impinging edge must interact. The fourth condition is that the disturbance waves must be reflected effectively. Finally, the disturbance waves must transmit upward, and induce some new vortex disturbance waves to generate in separating zone. The process can be expressed in Figure 3 .

With self-excited oscillation cavity, the first condition can be satisfied easily. To satisfy the second condition, at first, we assume that the function of the disturbance waves can be expressed as:

$$
\Psi_{1}(x, y, t)=\varphi(y) \exp [i(\alpha x-\omega t)]
$$

where: $\varphi(y)=\Phi_{R}+i \Phi_{1}$

$$
\begin{aligned}
& \alpha=\alpha_{R}+i \alpha_{1} \\
& \alpha_{R}=2 \pi / \lambda
\end{aligned}
$$

$\alpha_{R}$ represents the amount of waves

$\alpha_{1}$ represents space increasing factors

$\lambda$ represents the length of disturbance waves

$\Phi_{R}$ represents real part of $\varphi(y)$

$\Phi 1$ represents imaginary part of $\varphi(y)$

$$
\omega=\omega_{R}+i \omega_{i}
$$

$\omega_{R}=2 \pi / T$

$\omega_{i}$ represents development coefficient

If we assume $C_{S}=\frac{\varpi}{\alpha}=C_{R}+i C_{1}$

where:

$$
\begin{aligned}
& C_{R}=\frac{\lambda}{T}, C_{1}=\frac{\varpi_{1}}{\alpha} \\
& R_{e}=\frac{u_{0} L}{v}, \frac{U}{u_{0}}=\bar{U}, \frac{y}{L}=\bar{y}, \alpha L=\bar{\alpha}, \frac{C_{S}}{U}=\overline{C_{S}}, \frac{\varphi}{u_{0} L}=\bar{\varphi}
\end{aligned}
$$

Then, Equation (1) can be expressed as:

$$
\begin{aligned}
& \left(\overline{U-\overline{C_{S}}}\right)\left(\varphi^{\prime \prime}-\overline{\alpha^{2}} \bar{\varphi}\right)-\frac{d^{2} \bar{U}}{d y^{2}} \bar{\varphi} \\
& =-\frac{i}{\bar{\alpha} R_{e}}\left(\overline{\varphi^{(4)}}-2 \overline{\alpha^{2}} \overline{\varphi^{\prime \prime}}+\overline{\alpha^{4}} \bar{\varphi}\right)
\end{aligned}
$$

When $y \rightarrow \pm R, \varphi=\varphi^{\prime \prime}=0$.

And the frequency of disturbance waves can be expressed as: $S_{d}=f d / u_{0}$.

Based on all the equations above, we can find the relationship among space increasing factors $\alpha_{l}$, the amount of waves $\alpha_{R}$ and the frequency of disturbance waves $S_{d}$. Their relationship is shown in Figure 4.

Therefore, to satisfy the second condition, it is necessary that the rate of $L / d$ is in certain range ( $L$ represents the length of self-excited oscillation cavity, $d$ represents the diameter of the upstream nozzle).

When the disturbance waves transmit downward, they will impinge with impinging edge. So, the third condition can be satisfied easily.

To get self-excited oscillation pulsed jet, it is important to satisfy the fourth and fifth conditions. It means the disturbance waves generated in separating zone and the disturbance waves generated in impinging edge must satisfy some certain conditions. The transmitting speed of disturbance wave in shearing layer can be expressed in Equation (3).

$$
u^{\prime}(x)=\varphi^{\prime} e^{\alpha_{1} x} \exp \left[i a_{R} x-i \varpi t\right], o \leq x \leq L
$$

And the disturbance speed of disturbance waves generated in separating zone $(x=0)$ and the disturbance speed of disturbance waves generated in impinging edge $(x=L)$ can be expressed as Equations (4) and (5).

$$
\begin{gathered}
u^{\prime}(0)=\phi^{\prime} e^{i(-\varpi t)} \\
u^{\prime}(L)=\varphi^{\prime} e^{\alpha_{1} L} e^{i\left(\alpha_{R} L-\varpi t\right)}
\end{gathered}
$$

Under some certain conditions, the disturbance waves with largest speed in impinging edge can be gotten. Therefore, when they are reflected, we can get disturbance waves with the largest amplitude in separating zone. From Equations (4) and (5), we can get Equation (6).

$$
\alpha_{R} L=2 n \pi, n=1,2,3, \cdots
$$

where: $\alpha_{R}=\varpi / C_{s}, \omega=2 \pi f, f L / C s=n$.

If we take the influence of other factors into account, the factors including geometrical shape of impinging 


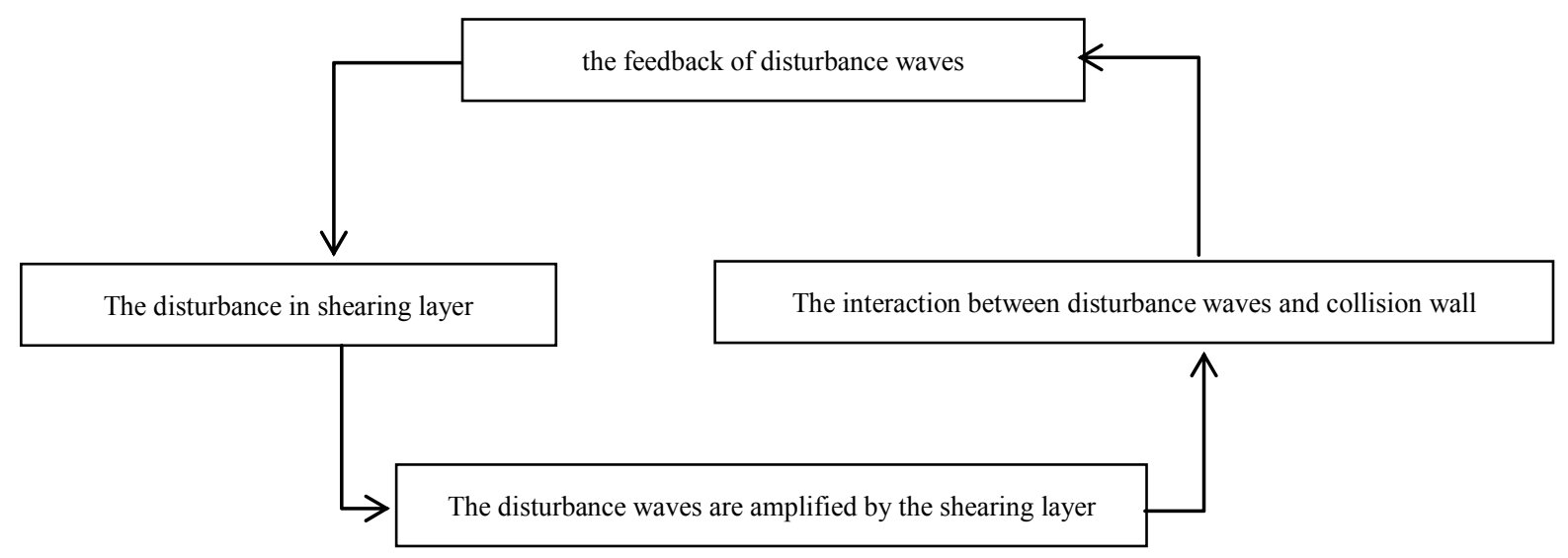

Figure 3. The transmitting process of disturbance waves in shearing layer.

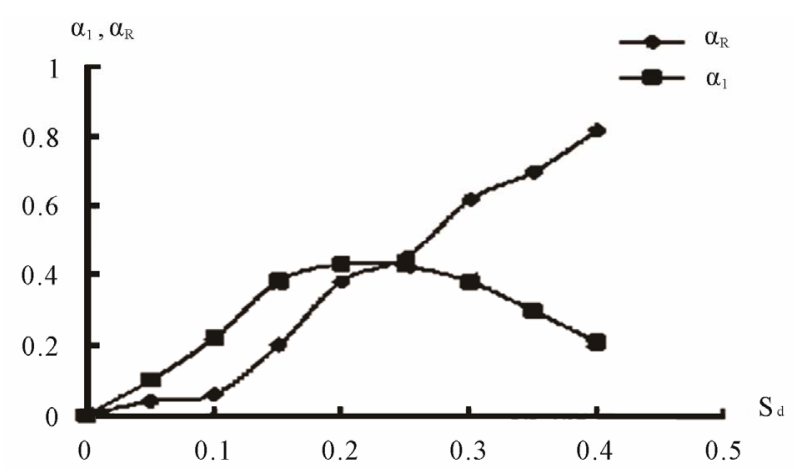

Figure 4. The relationship among $\alpha_{\mathrm{r}}, \alpha_{\mathrm{j}}$ and $S_{\mathrm{d}}$.

edge, the convolution of jet and the structure of self-excited oscillation cavity, the condition for effective feedback can be expressed in Equation (7).

$$
\frac{f L}{C_{s}}+\frac{\gamma}{2 \pi}=n
$$

In Equation (7), $\gamma$ represents lag phase angle of disturbance wave reflected.

Then

$$
S_{L}=f L / u_{0}=\frac{C_{s}}{u_{0}}\left(n-\frac{\gamma}{2 \pi}\right), n=1,2,3, \cdots
$$

So, to get effective reflection waves, the phase difference of disturbance waves in separating zone and impinging edge should be equal to $2 n \pi$.

\section{The Influence of Geometrical Shape of Impinging Edge on Self-Excited Oscillation Pulsed Jet [6]}

With impinging edge, the disturbance waves can be reflected effectively. However, different geometrical shape of impinging edges can generate disturbance waves with different amplitude. In order to study the influence of geometrical shape of impinging edge on self-excited oscillation pulsed jet, we adopt two-dimension vortex line model. At first, we presume the water flows un-rotationally. It means we ignore the size of vortex. According to the equation $z=x+j y$, we can get the Equation (9)

$$
W_{P}(z)=u_{p}-i v_{p}
$$

And in $x-y$ plane, the equation of motion of $J$ point vortex can be expressed as:

$$
u_{p}-i v_{p}=\frac{1}{Q_{0}} d \lambda_{p} / d z+\frac{i}{2 \pi} \sum_{j=1}^{M} \frac{\Gamma_{p j}}{z-z_{j}}+\frac{i}{2 \pi} \sum_{j=1}^{M} \frac{\Gamma_{p j}}{z-\frac{1}{\overline{z_{j}}}}
$$

where: $\lambda_{p}$ represents the plane with conformal transformation $\Gamma_{P I}$ represents the strength of No. I point vortex $\bar{Z}_{j}-Z_{j}$ represents the conjugate complex number pf $Z_{j}$.

To get fit geometrical shape of impinging edges, we study three types of impinging edges. The first shape of impinging edge is pyramidal face. The second is oval face. The third is plane. After calculation, the results are shown in Figures 5-7.

From Figures 5-7, it is obvious that, if we choose the geometrical shape of impinging edge as pyramidal face, the effect of self-excited oscillation pulsed jet is best.

\section{Experimental Study $[7,8]$}

\subsection{Measuring the Pressure of Different Points in Self-Excited Oscillation Cavity}

In order to verify the effectiveness of nozzle, the pressure of different points in oscillation cavity is measured. The points chosen are shown in Figure 8.

And the pressure waves of different points are shown in Figure 9.

From Figures $\mathbf{8}$ and $\mathbf{9}$, we can see, in the zone where points 1, 2, 3 are located, which is named as static zone, the pressure is small, and the amplitude of waves is small, too. But in the zone where points 5, 6 are located, which is named collision zone, the amplitude of waves is very big. And in the zone where point 4 is located, which is 


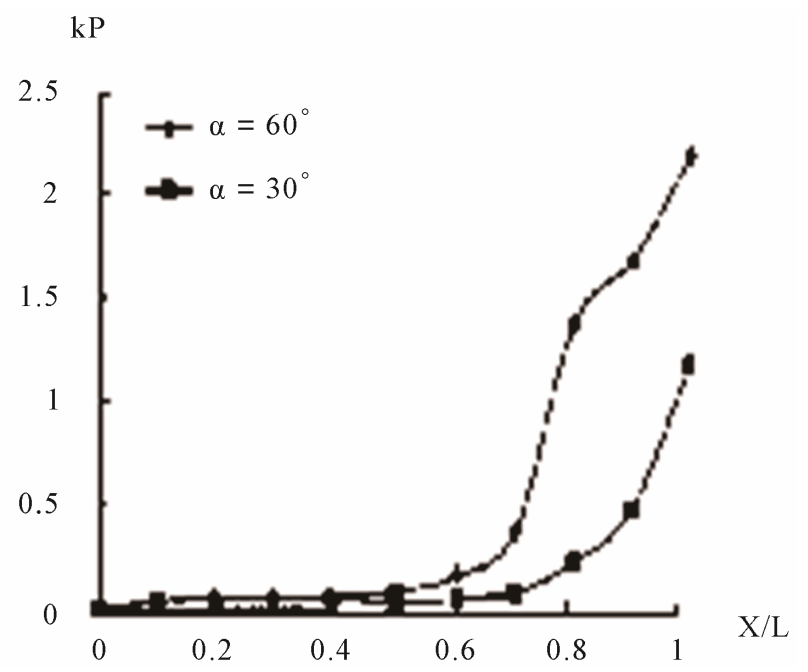

Figure 5. The influence of pyramidal face on self-excited oscillation pulsed jet.

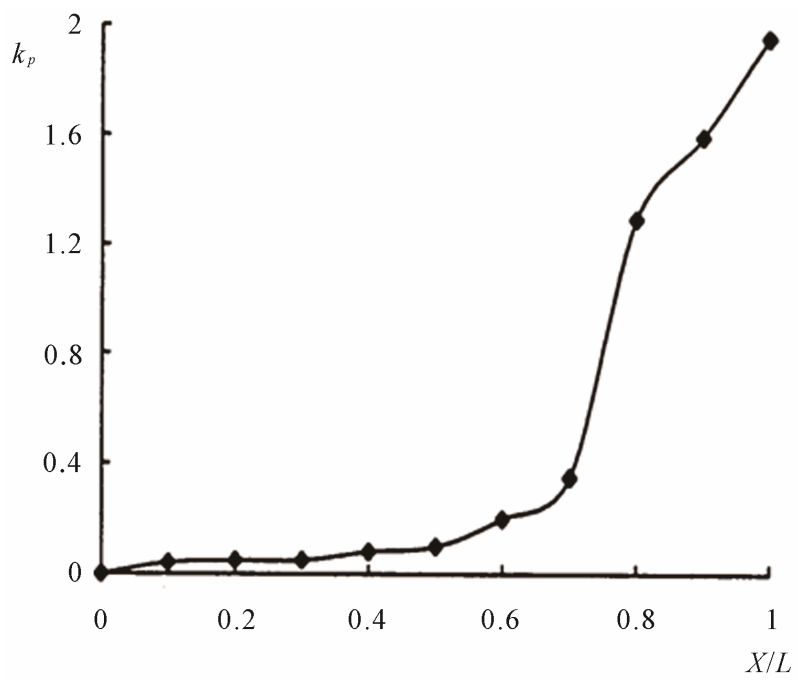

Figure 6. The influence of plane on self-excited oscillation pulsed jet.

named separating zone, the pressure of waves begins to be amplified. When disturbance waves and impinging edge interact, disturbance waves are reflected. Then the disturbance waves transmit upward. With the action of the disturbance waves, some new vortex waves which are periodic are generated near exit of upstream nozzle. If the phase position of disturbance waves which transmit upward and that of disturbance waves which transmit downward is same, the disturbance waves will be added repeatedly. From Figure 9, it is obvious that, the frequency of unstable waves which are generated at the beginning is higher than that which are generated later. The phase position of waves with different frequencies is not the same. So low-frequency waves can make shearing layer located in exit of upstream nozzle transform, and a series of high-frequency unstable waves combine to-

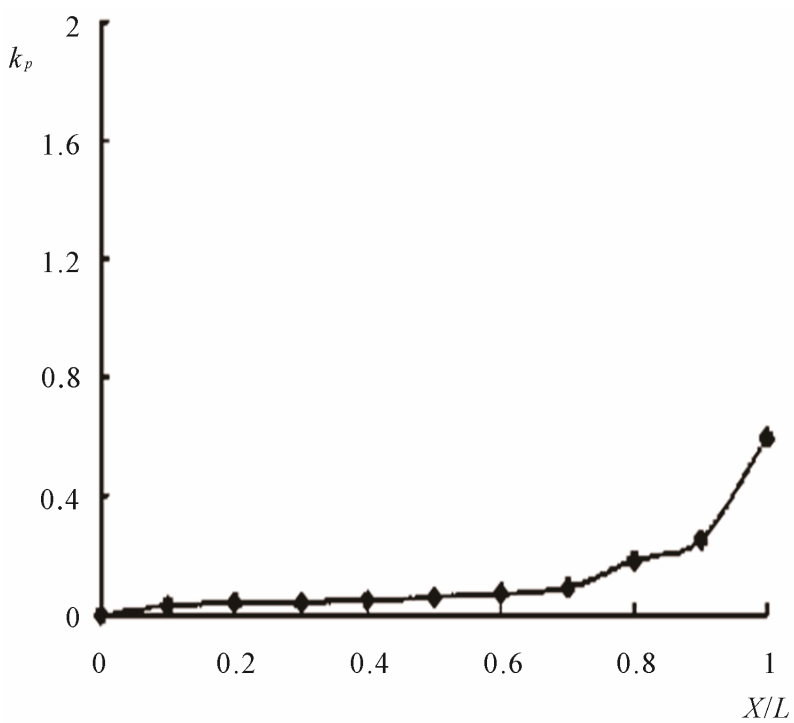

Figure 7. The influence of oval face on self-excited oscillation pulsed jet.

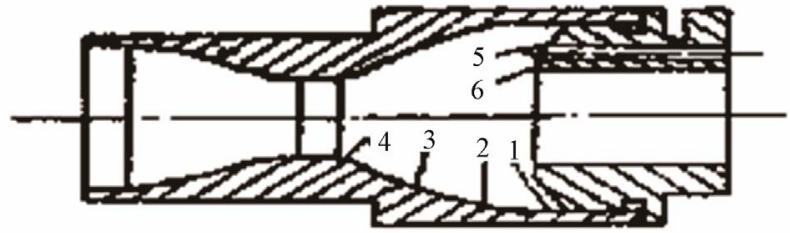

Figure 8. The points chosen for measuring.

gether. When they transmit downward, high-frequency unstable waves vanish quickly. And low-frequency waves are remained.

\subsection{The Experimental Study of Non-Submerged Jet Property}

We know different geometrical parameters of SEOPJN and hydraulic parameters are corresponding to different jet properties. In order to find optimum parameters, some experiments are made. And the experimental results are shown in Figures 10 and 11.

From Figures 10, 11, some conclusions can be drawn:

1) The pressure of jet is influenced by some factors including the rate of $L / d$ ( $L$ represents the length of cavity, $d$ represents the diameter of upstream nozzle), the rate of diameters of upstream nozzle and the diameter of downstream nozzle, the shape of impinging edge, the shape of cavity, etc.. If the rate of $\mathrm{L} / \mathrm{d}$ is too small, it is very difficult to form a stable shearing layer. But if the rate of $L / d$ is too large, the disturbance waves reflected can not induce the generation of new vortex waves. So, choosing suitable rate of $L / d$ is very important. In addition, the shape of impinging edge and the shape of cavity will influence the peak of pressure of jet.

2) From frequency Figure 11, we know that, the 


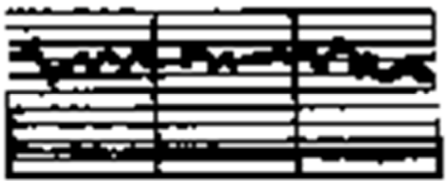

(a) The wave of the first point

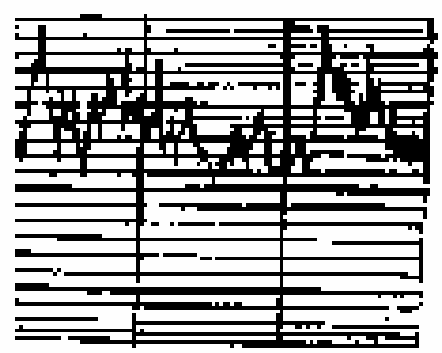

(d) The wave of the forth point

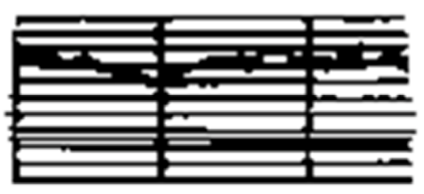

(b) The wave of the second point

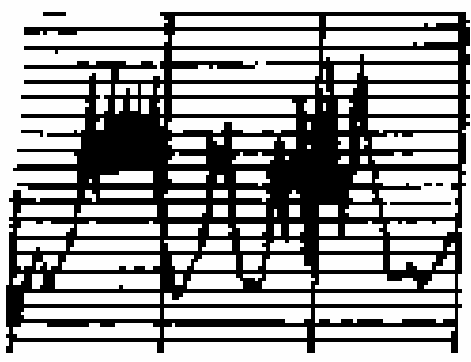

(e) The wave of the fifth point

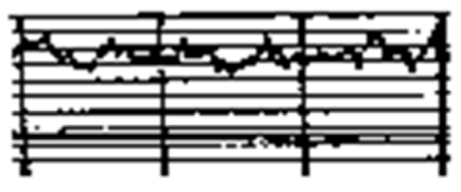

(c) The wave of the third point

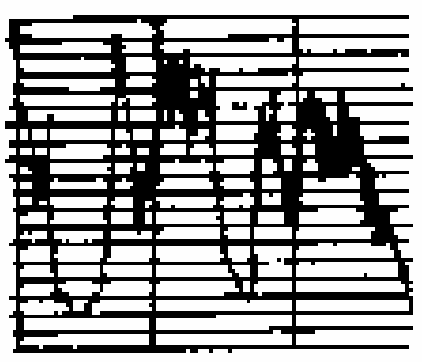

(f) The wave of the sixth point

Figure 9. The waves of different measuring points.

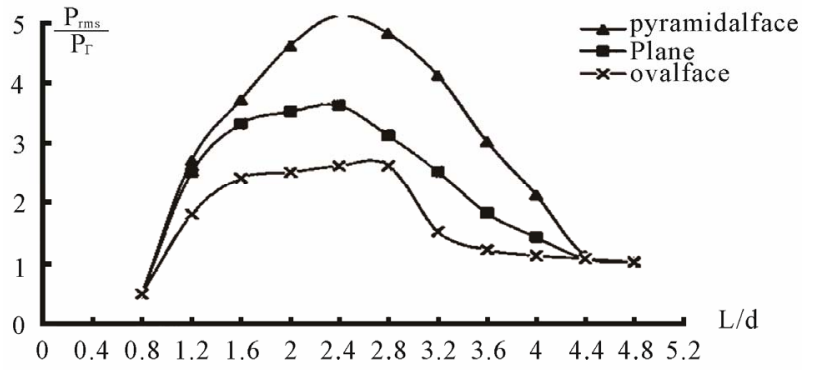

Figure 10. The relationship between the root-mean-square of pressure and rate of $L / d$.

waves of different frequencies are responding to different amplitude. But the waves whose frequencies approximate natural frequency of resonant cavity carry the largest amplitude. This shows that the waves with different frequency are amplified with various rates. So, it is understandable that only those disturbance waves whose frequencies are in certain range are amplified effectively. Especially those waves whose frequencies approximate natural frequency of resonant chamber are amplified greatly.

3) During the process of generating self-excited oscillation pulsed jet, the axial center pressure of jet fluctuates strongly, sometimes the pressure is positive, and sometimes it is minus. With it, a lot of air bubble and vaporous bubble are formed. As a result, cavitation can be generated.

4) With the increase of pressure of pump, the fluctuation of self-excited oscillation pulsed jet decreases, but the oscillation frequency increases.

\subsection{The Experimental Study of Submerged Water Jet Property}

To get submerged water jet property, some experiments are done. The experimental results are shown in Figures $12,13$.

From Figures 10 and 13, there are some differences between submerged water jet and non-submerged jet. To non-submerged water jet, when rate of $l / d=2.4$, the effect of jet is the best. But to submerged water jet, when rate of $l / d=3.6$, the effect of jet is the best. The reason for it is that, with submerged water jet, the water jet coming from nozzle will exchange momentum with water surrounding jet.

From Figure 14, we can know, under the same condition, the peak pressure of self-excited oscillation pulsed jet is $1.7-2.5$ times of the pressure of continuous jet. In addition, the more intensive the pressure fluctuates, the larger the effective standoff distance is. The reason for it is that, with the increase of fluctuation, cavitation is generated. As a result, a lot of vortex forms in interface between jet and water surrounding jet. This means the equivalent viscous coefficient of the shearing layer decreases. Therefore, the effective standoff distance is increased.

\subsection{The Comparison of Scouring Effect between Self-Excited Oscillation Pulsed Water Jet and Continuous Water Jet}

To compare the scouring effect, some sandstones whose compression strength is $30 \mathrm{MPa}$ are chosen. At first, take out two sandstones, put them at a place which is away from the exit of nozzle at a fixed distance. And the pump pressure is equal to $10 \mathrm{MPa}$. One of them is impacted with self-excited oscillation pulsed jet. Another is impacted by continuous jet. After 60 seconds, the erosion volume of each sandstones is measured by using the means of filling ceresin wax. Record the results. Then, take out another two sandstones, and choose another 


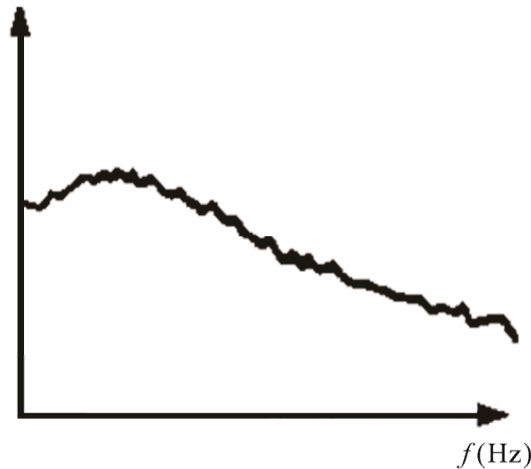

(a) $L / d=0, P_{0}=8 \mathrm{MPa}$

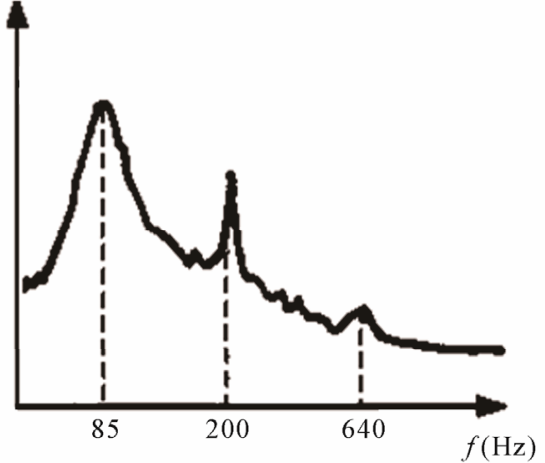

(b) $L / d=2.4, P_{0}=8 \mathrm{Mpa}$

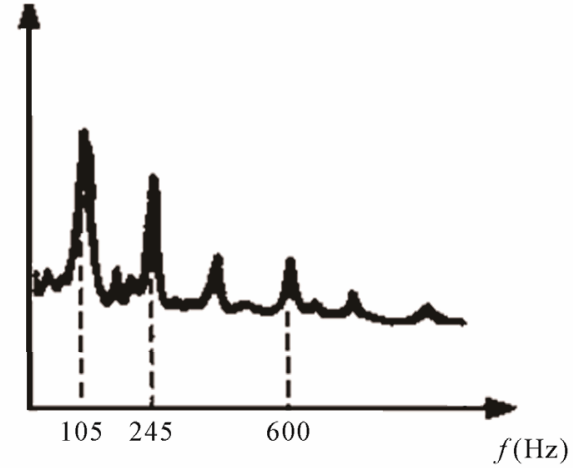

(c) $L / d=2.4, P_{0}=15 \mathrm{MPa}$

Figure 11. The frequency figure of axial center pressure of jet.

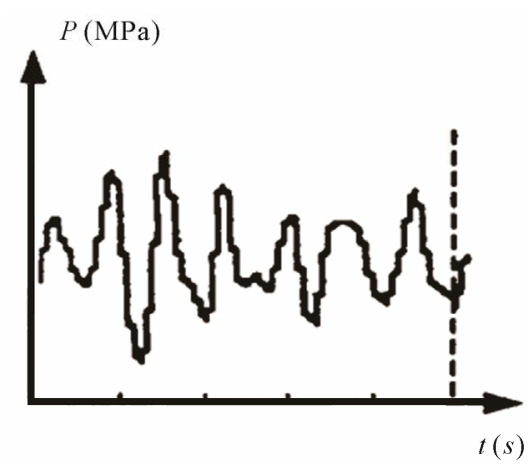

(a) $P_{0}=15 \mathrm{MPa}, L_{T} / d=4$

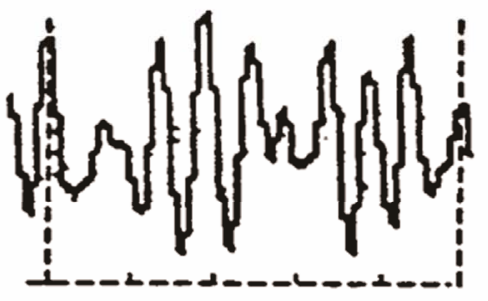

(b) $P_{0}=15 \mathrm{MPa}, L_{T} / d=10$

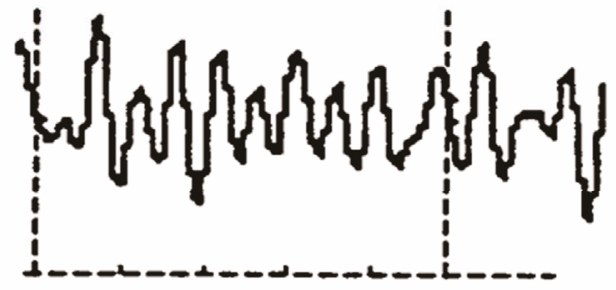

(c) $P_{0}=15 \mathrm{MPa}, L_{T} / d=14$

Figure 12. The waves of axial dynamic pressure of submerged water jet with different standoff distance.

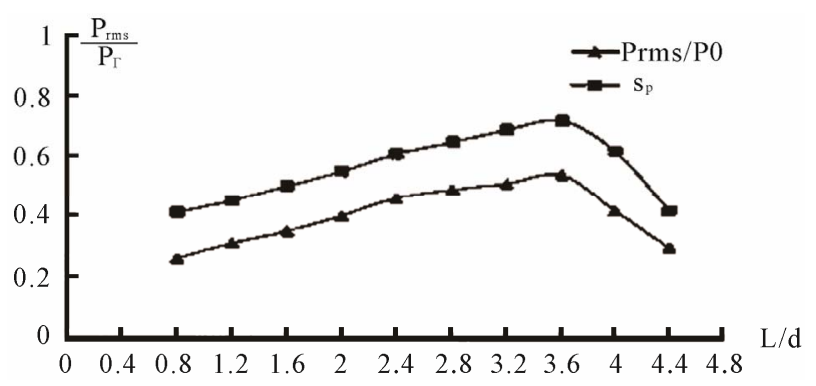

Figure 13. The relationship between the root-mean-square of pressure of submerged water jet and rate of $L / d$.

distance, then repeat the above process. With several times, the results can be shown in Figure 15.

From Figure 15, we can see, the volume erosion speed of self-excited oscillation pulsed jet is 4 times of that of continuous jet.

From Figure 16, we can see, with the external pressure of jet increasing, the volume erosion speed of both self-excited oscillation pulsed jet and continuous jet decrease. However, the erosion effect of self-excited oscillation pulsed jet is always better than that of continuous jet.

\section{The Application Study of SEOPJN}

From the above, it is obvious that the self-excited oscilla- tion pulsed jet is better than continuous jet. In order to prove that the SEOPJN is effective in industry, some nozzles are designed. This kind of nozzle is installed in tricorn bits. The structure of this kind of nozzle is shown in Figure 17, and the structure of tricorn bits is shown in Figure 18.

A lot of tests in 16 oil fields of China, such as South and North of Xingjiang, Sengli, Daqing, Zhongyuan, Chuandong oil fields etc., have been shown that the oscillating pressure in the well bottom is very large, and it can form great wall pressure potential and widen the influence region of the cross flow. Comparing with continuous jet, the peak of pressure of self-excited oscillation pulsed jet increases by $1.7-2.5$ times, the erosion volume increases by $1.5-2.0$ times, and the effective distance increases by 2 - 4 times. With the kind of nozzles, under the same condition, the exploring speed of bits increases by $8-77$ percent, and footage of each bit increases by $6.7-44.1$ percents. The reason for it is that, with SEOPJN, the water hammer effect is generated. To continuous jet, the energy $\mathrm{E}=1 / 2 \mathrm{mv}^{2}$, but to self-excited oscillation pulsed jet, the energy $E=\rho c v$,

Where: $v$ represents the speed of jet

$m$ represents the mass of water

$\rho$ represents density of water 


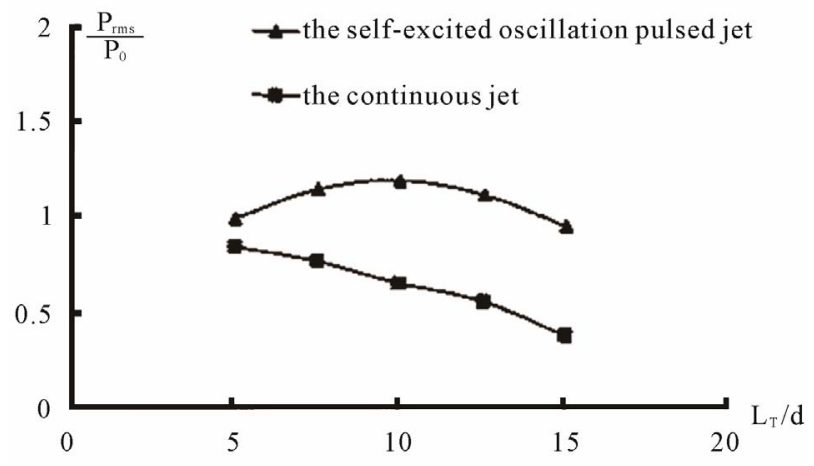

Figure 14. The comparison of pressure between self-excited oscillation pulsed submerged water jet and continuous submerged water jet.

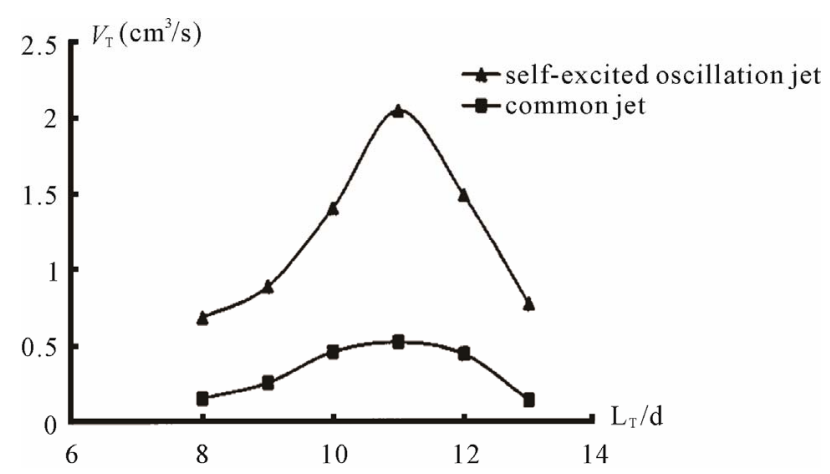

Figure 15. The comparison of volume erosion speed between non-submerged self-excited oscillation pulsed jet and non-submerged continuous jet.

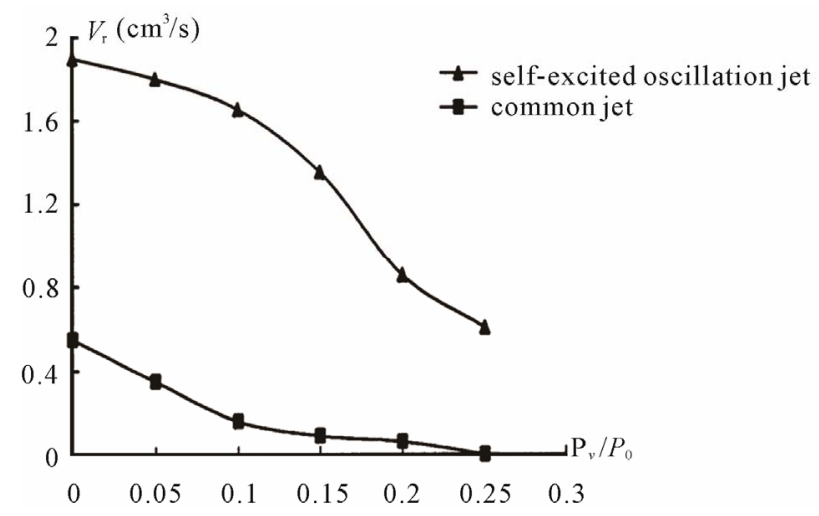

Figure 16. The comparison of volume erosion speed between non-submerged self-excited oscillation pulsed jet and non-submerged continuous jet when the external pressure of jet increases.

$c$ represents the speed of sound transmitting through water $(c=1400 \mathrm{~m} / \mathrm{s}, c>>v)$

The applications of SEOPJN here in China are marvelously successful and bring remarkable economical benefit to the state. The authors were awarded the national science and technology development second-rate

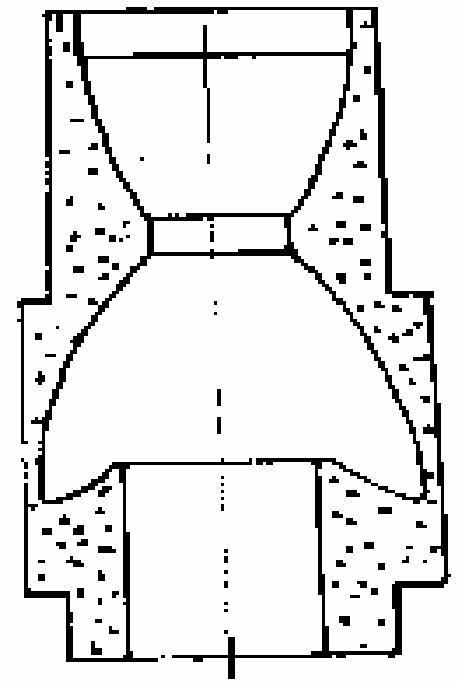

Figure 17. The structure of nozzle.

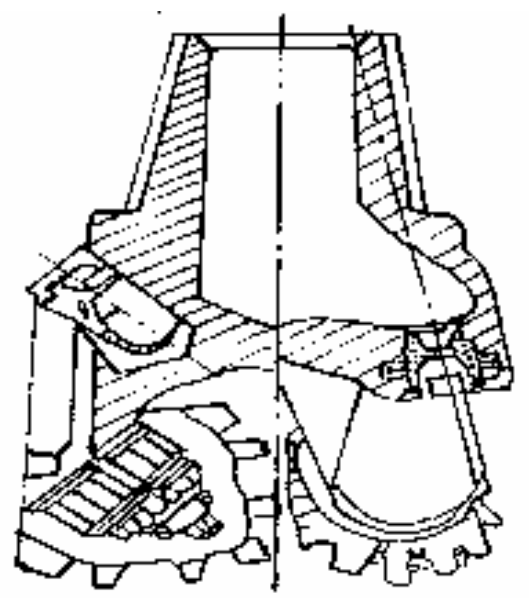

Figure 18. The structure of tricorn bits.

prize in 1992.

\section{Industrial Test of the Pipeline Pulsed Flow Generator for Natural Gas Pipeline [9]}

In recent years, many researchers have done a lot of studies in order to enhance the oil and natural gas pipeline transportation capacity and reduce pressure loss. Some suggestions to reduce the pressure loss during the transportation go through the help of the pulsed flow or wall vibration, and this has aroused people's interest. However, there has not been any industrial test report about this new proposal so far. The authors of this paper have done an industrial test of the Pipeline Pulsed Flow Generator (PPFG) at one of our country's oilfields.

\subsection{Installation}

The Pipeline Pulsing Flow Generator is built based on 
the theories of aerodynamics. When it is properly installed on the oil or natural gas pipeline, it can change the continuous flow into pulse jet without the help of any other auxiliary devices. The pressure increases accordingly at the exit of the generator. The sketch of the PPFG on natural gas transmission lines in Figure 19.

\subsection{Industrial Test}

A Pipeline Pulsing Flow Generator (natural gas pressure: $0.90 \mathrm{MPa}$; output flow rate: $12000-13000 \mathrm{~m}^{3} / \mathrm{d}$ ) had been installed at one of our country's oilfields for experiment since Oct. 2006. The test data are as follows: pressure at the wellhead: $(0.9-1.1) \mathrm{MPa}$; inside diameter of the pipeline: $75 \mathrm{~mm}$; the distance between the monitoring point and the wellhead: $1.2 \mathrm{~km}$. Electromagnetic fluid meter (model: Intelligent Precession fluid meter TDS$80 \mathrm{~B})$ are installed both at the monitoring points and the wellhead. The terminal of the pipeline is a boiler room into which two electromagnetic fluid meters have already been installed. Before the gas goes into the boiler room, first it is led into a surge tank (pressure: $0.15 \mathrm{MPa}$ ). A vent pipe is installed at the end of the pipeline for ventilation or other functions when the gas volume is too much

The test has employed two systems: one is conventional system (System 1 for short); the other is to install the Pipeline Pulsing Flow Generator at the bypass of the main gas pipeline (System 2 for short). Either of the two systems can shift into the other easily through a gate valve. At the beginning, System 1 is used for three days and nights and pressure and flow rate are measured respectively at the wellhead, the monitoring point and the boiler room. Then System 2 is employed for another three days and nights and pressure and flow rate are also measured respectively at the wellhead, the monitoring point and the boiler room. After many repeated tests, it is found that System 2 has the following features (the test data are repeatable):

1) When the Pipeline Pulsing Flow Generator produces are in operation, a clear whistling sound of about 40 to 60 decibels is produced. This sound and it causing vibration make the research personnel present feel comfortable. The clear the sound is, the better people may feel. This is an important feature of the device. The oscillating frequency of the Pipeline Pulsing Flow Generator is around tens of Hertz, and when people with hand to touch pipeline, they can feel the mild vibration.

2) Under the condition of constant pressure at the monitoring point (the pressure loss is $0.07 \mathrm{MPa}$ ), when the gas volume increases respectively from $635 \mathrm{~m}^{3} / \mathrm{h}$ and $520\left(\mathrm{~m}^{3} / \mathrm{h}\right)$ in System 1 to $1150 \mathrm{~m}^{3} / \mathrm{h}$ and $1180 \mathrm{~m}^{3} / \mathrm{h}$ $\left(1850 \mathrm{~m}^{3} / \mathrm{h}\right)$ in System 2, the tens of thousand of test data we get from several years' study has demonstrated a good repeatability. Details can be seen in Table 1.

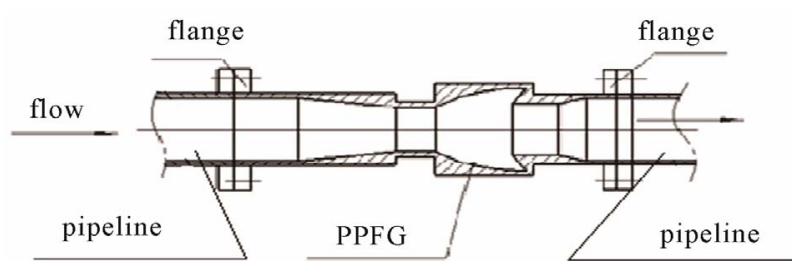

Figure 19. The sketch of the PPFG on natural gas transmission.

Table 1. Industrial test data.

\begin{tabular}{cccc}
\hline items & Volume $\left(\mathrm{m}^{3} / \mathrm{h}\right)$ & Volume $\left(\mathrm{m}^{3} / \mathrm{h}\right)$ & Volume $\left(\mathrm{m}^{3} / \mathrm{h}\right)$ \\
\hline System 1 & 635 & 520 & 520 \\
System 2 & 1150 & 1180 & 1850 \\
\hline
\end{tabular}

The Pipeline Pulsing Flow Generator has shown its great ability in drag reduction and gas transmission improvement. Several minutes after the plug-in of the Pipeline Pulsing Flow Generator, the gas transmission volume begins to increase, and the pressures at the portal tract in the gas gathering station and at the origin of the pipeline both begin to decrease.

\subsection{Several Explanations}

1) The test data obtained are not highly precise because of the shortage of funds and poor test skill.

2) The fact that the Pipeline Pulsing Flow Generator is installed not on the main line but on the bypass has a huge negative impact on its ability to reduce drag and to improve gas transmission.

3) The Pipeline Pulsing Flow Generator, after several years' operation, is still in good shape and no leakage has ever been detected. When it is uninstalled, it is dustless and as brand new as it was before.

4) The Pipeline Pulsing Flow Generator functions well in reducing drag and improving gas transmission when it is working under the designed situation (with proper pressure and gas volume) or so ( $\pm 15 \%$ around the designed situation). However, the actual working condition changes a lot, and it always deviates from the designed situation. Therefore, we have to process the large amount of data obtained.

5) Test shows that the Pipeline Pulsing Flow Generator is not only applicable to gas transmission, but also to the transmission of crude oil and refined oil. It also functions very well in reducing drag and improving transmission.

\section{REFERENCES}

[1] Z. F. Liao and D. S. Huang, "Nozzle Device for the Self-Excited Oscillation of a Jet," Proceedings of the 8th International Symposium on Jet Cutting Technology, Durham, August 1986, pp. 195-201. 
[2] Z. F. Liao and C. L. Tang, "Theoretical Analysis and Experimental Study of the Pulsed Jet Device," Proceeding of 4th US Water Jet Conference, Berkeley, 26-28 August 1987, pp. 27-34.

[3] Z. F. Liao, et al., "Theory and Experimental Study of the Self-Excited Oscillation Pulsed Jet Nozzle," Chinese Journal Mechanical Engineering, Vol. 16, No. 4, 2003, pp. 379-383. doi:10.3901/CJME.2003.04.379

[4] Z. F. Liao, C. L. Tang and G. H. Wang, "A New Type of Nozzle for Oil-Well Drill Bits," Proceedings 10th International Symposium on Jet Cutting Technology, Netherlands, 31 October-2 November 1990, pp. 135-144.

[5] Z. F. Liao and C. L. Tang, "Pulsed Jet Nozzle for Oil-Well Jetting Drilling," Proceedings of the 7th American Water Jet Conference, Seattle, 28-31 August 1993, pp. 537-548.
[6] C. L. Tang, X. H. Li and Z. F. Liao, "The Study of Oscillation Jet Nozzle with Flow control Oscillator," Proceedings of the 9th American Water Jet Conference, Dearborn, 23-26 August 1997, pp. 27-34.

[7] C. L. Tang and Z. F. Liao, "The Application of the Airlift and the Self-Excited Oscillation Pulsed on Exploiting Beach Placer," 14th International Conference on Jetting Cutting Technology, Brugge, 21-23 September 1998, pp. 375-386.

[8] C. L. Tang and Z. F. Liao, "The Theory and Experimental Study of Self-Excited Oscillation Pulsed Jet Nozzle," Journal of China Coal Society, Vol. 12, No. 1, 1989, pp. 90-100.

[9] Z. F. Liao, D. S. Chen and Y. F. Kang, "The Pipeline Pulsing Flow Generator," China Patent No. ZL200510057466.6, 2006. 